\title{
Otimização do processo produtivo de uma linha de panificação
}

DOI: https://doi.org/10.35168/2176-896X.UTP.Tuiuti.2020.Vol6.N61.pp28-67

\section{Arion Rebeca Pereira}

Universidade Tuiuti do Paraná, PR, Brasil. arionpr@gmail.com

\section{Diego Sartori Felkl}

Universidade Tuiuti do Paraná, PR, Brasil. diegosfelkl@gmail.com

\section{Rodrigo Ramos Alves}

Universidade Tuiuti do Paraná, PR, Brasil. rodrigo.alves@utp.br

Miguelangelo Geimba de Lima Universidade Tuiuti do Paraná, PR, Brasil. miguelangelo.lima@utp.br 


\section{Otimização do processo produtivo de uma linha de panificação}

\section{Resumo}

Para manter a empresa competitiva no mercado com produtos de qualidade necessita-se de eficiência nos processos produtivos, evitando perdas, otimizando o uso de recursos e, ainda, aumentando a capacidade produtiva. Neste sentido a finalidade deste trabalho é a otimização do processo produtivo de uma linha de panificação de uma empresa localizada em Araucária - PR. Com o uso de ferramentas de análise e solução de problemas, promoveu-se uma avaliação do processo e ações de melhoria. Através da estruturação de dados foi possível observar que a produção melhorou sua produtividade significativamente sem que houvessem custos extras para mudanças. As sugestões de melhorias foram testadas por uma semana, e os resultados foram satisfatórios.

Palavras-chave: Eficiência. Produtividade. Melhoria nos processos. 


\section{Optimization of production process of a bakery line}

\section{Abstract}

To keep the company competitive in the market with quality products need to efficiency in production processes, avoiding losses, optimizing the use of resources and also increasing production capacity. In this sense the purpose of this work is the optimization of the production process of a bakery line for a company in Araucaria - PR. Using analysis and problem solving tools, an evaluation of the process and improvement actions were promoted. By structuring data it was observed that the production significantly improved its productivity without extra costs that had to change. The suggestions for improvements were tested for a week, and the results were satisfactory.

Keywords: Efficiency, Productivity, Process improvement. 


\section{Otimização do processo produtivo de uma linha de panificação}

\section{Introdução}

Em um ambiente de constantes mudanças, há um esforço necessário nas fábricas para que as mesmas sejam eficazes nos seus sistemas de produção e capazes de produzir a baixos custos (HANSEN, 2006).

Neste contexto, há a necessidade das firmas em explorarem e implantarem sistemas e modelos de produção que tenham um resultado satisfatório do ponto de vista de custos, qualidade, tempo, flexibilidade e inovação (ANTUNES et al, 2008).

Novas tecnologias surgem como fatores competitivos, onde a inovação tanto em nível de produto como de processo torna-se essencial para que as empresas mantenham sua fatia de mercado e estejam aptas a explorar novos mercados. Ainda mencionam que os avanços tecnológicos em diversas áreas se desenvolvem de forma cada vez mais rápida, visando aperfeiçoar a produtividade do setor industrial, bem como em outros setores.

A base para o desenvolvimento do presente trabalho é a unidade industrial de panificação em Araucária - PR. O foco é a aplicação de ferramentas de análise e solução de problemas na linha de produção de pães da fábrica, onde, em uma análise prévia, percebeu-se oportunidades de melhoria nos processos, sendo possível aumentar a capacidade produtiva, otimizar o uso de recursos e evitar perdas.

O objetivo deste trabalho é a otimização do processo de produção de pães da empresa analisada. Com este objetivo buscou-se a diminuição mão de obra envolvida no processo, a adequação do trabalho com a redução do tempo de utilização e o aumento da eficiência da máquina modeladora de pães, a padronização dos tempos de atividade e o aumento da capacidade produtiva por colaborador. 


\section{Otimização do processo produtivo de uma linha de panificação}

\section{Material e métodos}

Nesta parte serão abordadas a fundamentação teórica, a descrição da empresa em estudo e o procedimento metodológico utilizado neste trabalho.

\subsection{FUNDAMENTAÇÃO TEÓRICA}

\subsubsection{Sistemas de produção}

Os sistemas podem ser compreendidos como um grupo de componentes inter- relacionados que trabalham juntos rumo a uma meta comum recebendo insumos (entradas do sistema) e produzindo resultados (saídas do sistema), em um processo organizado de transformação (ANTUNES et al, 2008).

Para essa transformação, é necessário um processo que é visto como um fluxo de materiais, ou produto em um determinado tempo e espaço, para transformar matéria-prima em componentes e produtos semiacabados em produtos acabados. A figura 1 representa um modelo de sistema de produção.

Figura 1 - Modelo de sistema de produção

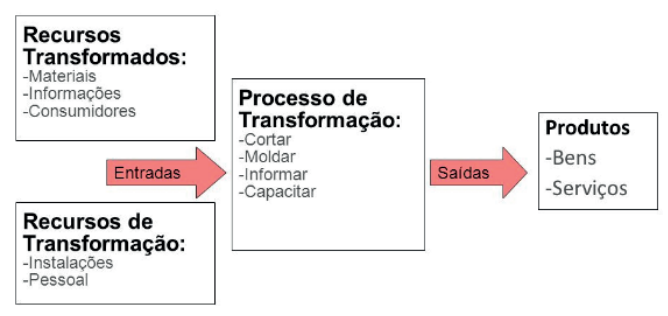

Fonte: adaptado de SLACK et al (1997). 


\section{Otimização do processo produtivo de uma linha de panificação}

\subsubsection{Tipos de Sistemas de Produção}

Moreira (1998) define os principais tipos de sistemas de produção, sendo a classificação tradicional, em função do fluxo do produto, agrupando os sistemas de produção em três grandes categorias:

a. Sistemas de produção contínua ou de fluxo em linha: apresentam sequência linear de fluxo e trabalham com produtos padronizados. Este tipo de sistema pode ser produção contínua propriamente dita e produção em massa.

b. Sistemas de produção intermitente: Este sistema pode ser por lote ou por encomenda.

c. Sistemas de produção de grandes projetos sem repetição: produto único, não há rigorosamente um fluxo do produto, existe uma sequência pré-determinada de atividades que deve ser seguida, com pouca ou nenhuma repetitividade.

Tubino (2007) discute de maneira mais ampla as classificações dos sistemas de produção, identifica o critério que serve de base para três delas:

a. Pelo grau de padronização: Podendo ser sistemas que produzem produtos padronizados e sistemas que produzem produtos sob medida.

b. Pelo tipo de operação: Podendo ser processos contínuos, processos discretos, processos repetitivos em massa, processos repetitivos em lote, processos por projeto.

c. Pela natureza do produto: Neste tipo pode ser manufatura de bens e prestador de serviços. 


\section{Otimização do processo produtivo de uma linha de panificação}

\subsubsection{Balanceamento de linha}

Para um correto desenvolvimento do processo produtivo dentro de uma linha de produção, se faz necessário um balanceamento dos postos de trabalho, de forma a otimizar a produção ali executada.

Tubino (2007) afirma que o balanceamento de células ou postos de trabalho possuem uma sequência lógica de ações dentro da linha de montagem, com os operadores dispostos nos postos de trabalhos, seguindo um conjunto de operações- padrão necessárias para montar o produto, ou realizar submontagens de componentes.

Para Fitzsimons e Fitzsimons (2004) o balanceamento consiste em equilibrar as operações dos postos de trabalho a partir das rotinas de operações sincronizadas dentro de um tempo ciclo capaz de atender uma demanda determinada, a partir da utilização de supermercados de abastecimento de linhas de montagem que utilizam layouts adequados a cada operação.

\subsubsection{Tempo de ciclo, normal e padrão}

O tempo de ciclo refere-se às etapas seguidas para completar um processo. A simplificação de ciclos de trabalho e a queda de barreiras entre etapas improdutivas no processo permitem que a qualidade total seja bem sucedida. O ciclo operacional permite a competição pelo tempo, o atendimento mais rápido do cliente, etapas de produção mais encadeadas entre si, queda de barreiras e obstáculos intermediários. Os conceitos de fabrica enxuta e Just in time são baseados no ciclo de tempo reduzido (CHIAVENATO, 2006). 


\section{Otimização do processo produtivo de uma linha de panificação}

\subsubsection{Ferramentas da qualidade}

As ferramentas da qualidade visam por meio do ataque à causa (processo), extinguir e coibir o aparecimento de problemas (efeitos) (OLIVEIRA et al, 2006).

A maioria das ferramentas da qualidade são de simples operação e o seu uso adequado garante a transformação dos dados estatísticos em ações de melhoria e correção de rumos. Apesar de atualmente haverem centenas de técnicas operacionais e gerenciais aceitas como ferramentas da qualidade descreve a existência de sete destas tecnologias, a saber: gráficos de estratificação, folhas de verificação, análise de Pareto, diagramas de Ishikawa, histogramas, diagramas de dispersão, gráficos de controle (ROCHA; GOMES, 1993).

a. Diagrama de Dispersão: Os diagramas de dispersão consistem em técnicas gráficas utilizadas para visualizar e também para analisar as relações entre duas variáveis. De modo geral, são usados para relacionar causa e efeito, como, por exemplo, o relacionamento entre velocidade de corte e rugosidade superficial em um processo de usinagem, composição de material e dureza, intensidade de iluminação de um ambiente e erros em inspeção visual, e outros (CARVALHO et al, 2012).

b. Cartas de Controle: Os gráficos (cartas) de controle são ferramentas para o monitoramento da variabilidade e para a avaliação da estabilidade de um processo. Verificar a estabilidade de um processo evita que haja um processo instável capaz de gerar produtos defeituosos, perda de produção, baixa qualidade, ou seja, resultar na perda da confiança do cliente. Existem dois tipos de causas para a variação: causas comuns ou aleatórias e as causas especiais ou assinaláveis (WERKEMA, 1995). O primeiro são aquelas variações provocadas por uma variabilidade natural do processo presentes em todos os processos. O segundo tipo de variação são aquelas que surgem esporadicamente. 


\section{Otimização do processo produtivo de uma linha de panificação}

c. Fluxograma: Quando se faz uma representação de um processo, normalmente observase que algumas rupturas identificadas já eram conhecidas e, portanto, tomadas ações paliativas para resolver problemas a curto prazo. O mapeamento de processo pode trazer benefícios na medida que oferece um conjunto de técnicas que podem representar de maneira simplificada as relações entre os vários processos de uma empresa (DORNELES; GASPARETO, 2015).

\subsection{A Empresa em estudo}

Este trabalho foi elaborada em uma empresa do ramo alimentício, localizada na região de Araucária - PR, que atua no setor privado e público, fornecendo aproximadamente 500 mil refeições por dia.

Seu quadro de colaboradores atualmente conta com 5 mil funcionários divididos em vários estados e vários setores, sendo um destes o setor de panificação, localizado na cidade de Araucária - PR, onde atuam 20 funcionários em 2 turnos de trabalho.

A demanda diária é suficiente para que os 2 turnos trabalhem de forma ininterrupta ao longo da jornada de trabalho, porém percebe-se que existe capacidade ociosa devido as falhas de operação e a não utilização de métricas de controle, as quais permitiriam a tomada de decisões estrategicamente mais eficientes.

\subsection{Procedimento metodológico}

Quanto à abordagem as pesquisas podem ser qualitativa e quantitativa. A pesquisa qualitativa não se preocupa com representatividade numérica, mas, sim, com o aprofundamento da compreensão 


\section{Otimização do processo produtivo de uma linha de panificação}

de um grupo social, de uma organização. Diferentemente da pesquisa qualitativa, os resultados da pesquisa quantitativa podem ser quantificados. Quanto aos procedimentos as pesquisas podem ser estudo de caso, pesquisa documental, pesquisa bibliográfica, levantamento, expost-facto, pesquisa participante, pesquisa-ação, pesquisa etnográfica, pesquisa fenomenológica e pesquisa experimental (MARCONI; LAKATOS, 2006).

A abordagem utilizada neste trabalho foi a quantitativa e quanto aos procedimentos foi um estudo de caso.

Neste sentido este trabalho consiste em aplicar conhecimentos e ferramentas técnicas para solução de problemas com o propósito de melhorar a eficiência produtiva de linha de produção de pães em uma indústria alimentícia.

Para possibilitar a análise dos dados e o alcance dos objetivos propostos, e visando a gestão de produção orientada a produtividade, este trabalho possui natureza de abordagem quantitativa. Como método de pesquisa foi utilizado o levantamento de dados in loco, com anotação de dados de produtividade e observações em linha de produção.

A primeira etapa do trabalho foi composta do levantamento de dados e elaboração de gráficos que permitam visualizar o processo atual, suas falhas e pontos de melhoria.

A coleta de dados foi feita in loco, diretamente na linha de produção, com o auxílio de cronômetros, folhas de processos e cronoanálise, baseadas em grandes grupos de amostras e medições. Após esta etapa foram calculados os tempos de produção, a capacidade produtiva dos recursos utilizados, tempos de ciclo e de operação e foram verificadas as possibilidades de balanceamento da linha e necessidades do projeto. A última parte compõe sugestões de melhorias e resultados pós testes. 


\section{Otimização do processo produtivo de uma linha de panificação}

\section{RESULTADOS E DISCUSSÃO}

Este trabalho foi realizado no setor de panificação, especificamente na linha de produção de pães, dentro de uma indústria de alimentos, situada Araucária - PR.

O mix de produção é composto de vários tipos de pães, porém, apesar da grande variedade existente, o setup da máquina modeladora é facilitado, pois a única mudança necessária ao equipamento é a substituição das facas de corte, processo que tem seu tempo estimado em 1 minuto.

Os recursos utilizados para produção possuem capacidade máxima e limitada de produção, ainda não mensuradas. A produção é do tipo puxada, com a existência de lotes, e, devido à natureza do negócio (contratos com quantidades específicas de fornecimento), a demanda é conhecida com um mês de antecedência.

\subsection{Fluxo de produção}

A primeira etapa do estudo consiste em conhecer o processo produtivo do setor estudado. $\mathrm{O}$ mapeamento do processo foi realizado conforme a figura 2 a seguir: 


\section{Otimização do processo produtivo de uma linha de panificação}

Figura 2 - Fluxograma do processo

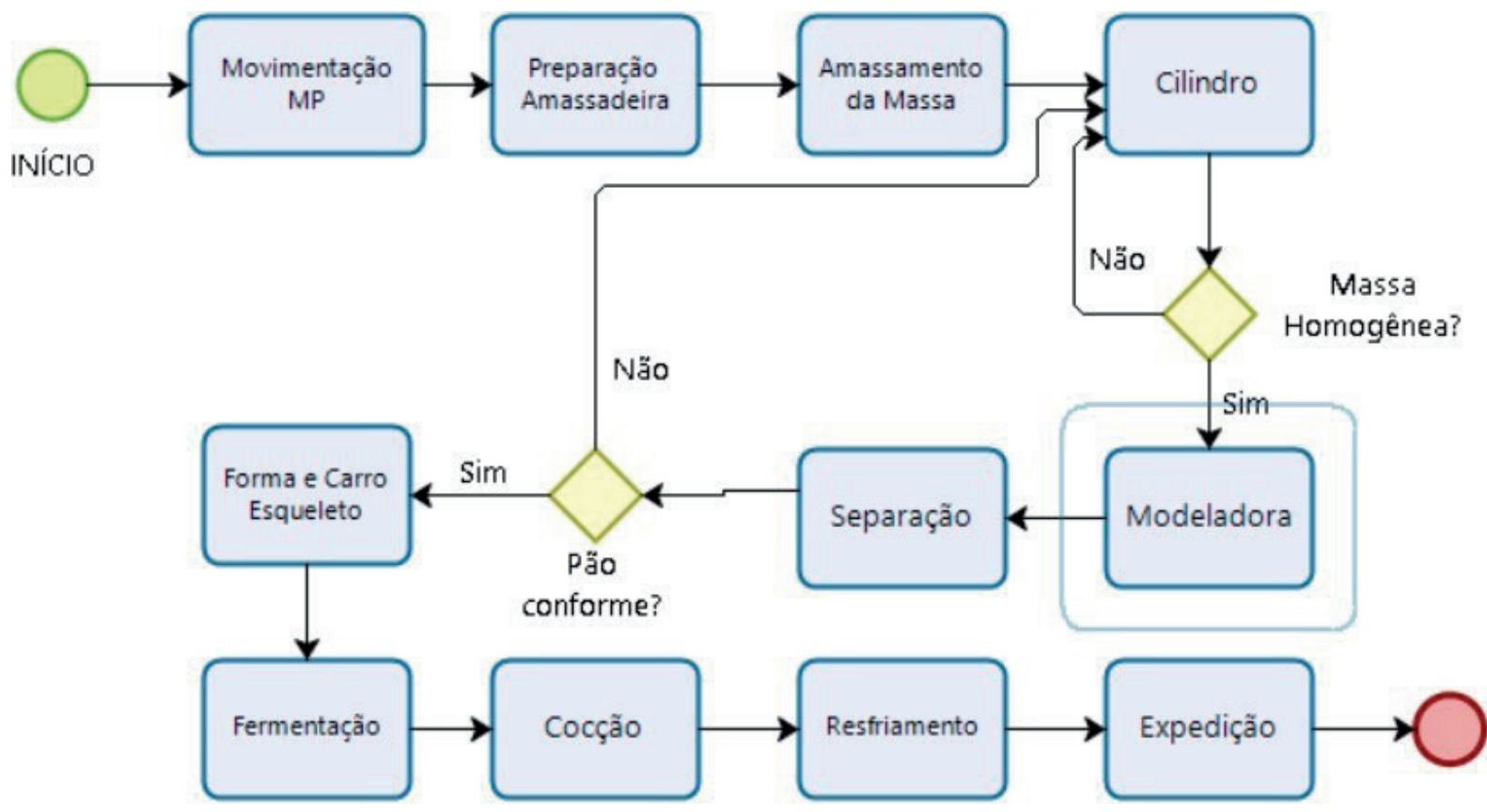

FIM

Fonte: Autores (2019).

\subsubsection{Movimentação de matéria prima}

A movimentação da matéria prima é feita do armazém diretamente para área produtiva com a utilização de paleteiras, por um percurso de 30 metros, sendo o material separado sempre no dia 


\section{Otimização do processo produtivo de uma linha de panificação}

anterior. Um operador é responsável por esta etapa, com o trigo separado em pallets e os outros insumos (gordura vegetal, ovo em pó, reforçador e corante) em sacos formando um Blend. Esta movimentação é realizada 2 vezes ao dia e pode ser considerada como o tempo de preparação.

\subsubsection{Abastecimento da amassadeira}

Esta etapa consiste no abastecimento manual das amassadeiras com os ingredientes necessário a fabricação do pão. Para uma receita padrão, com utilização da amassadeira para $160 \mathrm{Kg}$ são utilizados os seguintes ingredientes:
a) $100 \mathrm{Kg}$ de farinha de trigo
b) 401 de água
c) Mistura (ovo em pó, gordura vegetal, sal, açúcar e reforçador) $20 \mathrm{Kg}$.

\subsubsection{Amassamento da massa}

Esta operação é realizada de forma automática com a utilização das Amassadeiras, o tempo padrão é de 15 minutos por receita. Um tempo maior pode fazer com que a massa ultrapasse a temperatura ótima de produção, fazendo com o que produto fermente antes do tempo.

\subsubsection{Cilindro}

Etapa manual em que os operadores devem passar a massa através do conjunto de cilindros. Esta etapa tem como objetivo preparar a massa em mantas homogêneas com espessura padronizada para a utilização na máquina modeladora. 


\section{Otimização do processo produtivo de uma linha de panificação}

\subsubsection{Modelagem}

Nesta etapa a massa é modelada de acordo com a regulagem configurada para o equipamento. A manta de massa homogênea, retirada do processo anterior, é utilizada para alimentação do conjunto de modelagem, após a conformação da massa através do conjunto de cilindros.

\subsubsection{Separação}

Após a passagem da massa através dos cilindros de conformação e modelagem, o produto é transportado pela esteira transportadora. Nesta etapa o produto que apresenta a característica padrão de qualidade é separado por 4 operadores e posicionado em formas retangulares, cada forma possui capacidade para 80 pães. Após cheia, a forma é transferida para carros esqueletos, sendo que cada carro tem capacidade de 16 formas. O produto fora das especificações é separado e volta para masseira.

\subsubsection{Fermentação e crescimento}

Nesta etapa, após cheio ou parcialmente cheio, de acordo com o tamanho do lote a ser produzido, o carro é transferido para câmara de fermentação, local com temperatura e umidade controla. Esta câmara tem como objetivo acelerar o processo de crescimento do produto.

\subsubsection{Cocção}

Após o produto ter atingido o tamanho ideal, o carro é transferido para área de cocção onde é assado. O tempo padrão de cocção é de 20 minutos. Os fornos possuem timer e alarme com aviso sonoro de tempo. 


\section{Otimização do processo produtivo de uma linha de panificação}

\subsubsection{Resfriamento}

O processo de resfriamento tem como objetivo acelerar o resfriamento dos pães e consiste de 2 etapas. Na primeira os pães sofrem ventilação forçada, em que ventiladores são responsáveis jogar ar frio diretamente nos carros recém-saídos dos fornos. Na segunda etapa os pães ficam naturalmente resfriando em área pré- determinada.

\subsubsection{Expedição}

Este último processo é destinado a separação e empacotamento, realizado de acordo com a quantidade específica de cada cliente.

\subsection{Rotina de produção}

A rotina de produção se inicia com 1 mês de antecedência, no momento em que os cardápios dos contratos de fornecimento são montados. A partir deste momento é criado o mapa de produção, que contém a quantidade diária a ser produzida.

A demanda média é de 1.700 .000 pães por mês, com base nos meses de setembro, outubro, novembro e dezembro de 2018, portanto são produzidos diariamente aproximadamente 70 mil pães.

A figura 3 demonstra os postos de trabalho e a quantidade de operadores em cada posto. Também é possível verificar a existência de duas equipes de trabalho, com o primeiro turno em azul e o segundo turno em verde, o intervalo para refeições é representado pela cor laranja. Os números representam a quantidade de colaboradores por posto de trabalho. 


\section{Otimização do processo produtivo de uma linha de panificação}

Figura 3 - Distribuição da jornada e postos de trabalho

\begin{tabular}{|c|c|c|c|c|c|c|c|c|c|c|c|c|c|c|c|c|c|c|c|c|}
\hline \multicolumn{21}{|c|}{ PROCESSO ATUAL - Distribuição de postos de trabalho } \\
\hline Linha do Tempo & 06 às 07 & 07 às 08 & 08 ás 09 & 09 às 10 & 10 às 11 & 11 às 12 & 12 às 13 & 13 às 14 & 14 às 15 & 15 às 16 & 16 às 17 & 17 às 18 & 18 às 19 & 19 às 20 & 20 às 21 & 21 às 22 & 22 às 23 & 23 às 24 & 24 às 01 & 01 ás 02 \\
\hline Amassadeira & & 1 & 1 & 1 & 1 & & 1 & 1 & 1 & 1 & & 1 & & 1 & 1 & 1 & 1 & 1 & 1 & 1 \\
\hline Cilindro & & 2 & 2 & 2 & 2 & & 2 & 2 & 2 & 2 & & 2 & & 2 & 2 & 2 & 2 & 2 & 2 & 2 \\
\hline Abastecimento & & 1 & 1 & 1 & 1 & & 1 & 1 & 1 & 1 & & 1 & & 1 & 1 & 1 & 1 & 1 & 1 & 1 \\
\hline GA & & 4 & 4 & 4 & 4 & & 4 & 4 & 4 & 4 & & 4 & & 4 & 4 & 4 & 4 & 4 & 4 & 4 \\
\hline Fomo & & 1 & 1 & 1 & 1 & & 1 & 1 & 1 & 1 & & 1 & & 1 & 1 & 1 & 1 & 1 & 1 & 1 \\
\hline Raspagem & 1 & 1 & 1 & 1 & 1 & & 1 & 1 & 1 & & 1 & 1 & 1 & 1 & 1 & 1 & 1 & 1 & 1 & \\
\hline Total de MO & 1 & 10 & 10 & 10 & 10 & 0 & 10 & 10 & 10 & 9 & 1 & 10 & 1 & 10 & 10 & 10 & 10 & 10 & 10 & 9 \\
\hline \multirow[t]{3}{*}{ LEGENDA } & Equipe 1 & & & & & & & & & & & & & & & & & & & \\
\hline & Equipe 2 & & & & & & & & & & & & & & & & & & & \\
\hline & Intervalo & & & & & & & & & & & & & & & & & & & \\
\hline
\end{tabular}

Fonte: Autores (2019).

A linha de produção objeto do estudo, contém 20 colaboradores, divididos em 2 turnos de 8 horas, com turnos de 4 horas aos sábados. A primeira equipe inicia a jornada de trabalho às $07 \mathrm{~h} 00 \mathrm{~min}$ com intervalo entre às $11 \mathrm{~h} 30 \mathrm{~min}$ e $12 \mathrm{~h} 30 \mathrm{~min}$, e término às $16 \mathrm{~h} 00 \mathrm{~min}$. A segunda equipe inicia os trabalhos às $17 \mathrm{~h} 00 \mathrm{~min}$, com intervalo entre às $18 \mathrm{~h} 00 \mathrm{~min}$ e $19 \mathrm{~h} 00 \mathrm{~min}$, e tem seu término $01 \mathrm{~h} 45 \mathrm{~min}$.

\subsection{Estudo de tempos, métodos e produtividade}

Com o objetivo de realizar o projeto de melhoria, seguir as diretrizes estabelecidas pela empresa, e após mapeamento das principais atividades realizadas durante a produção de pães, foi iniciado o planejamento para realização dos estudos de tempos e métodos praticados.

Nesta etapa foi mensurada a produtividade atual da produção e também os métodos praticados para cada recurso e processos anteriormente levantados. Em atividades manuais, em que o ritmo e 


\section{Otimização do processo produtivo de uma linha de panificação}

produtividade da operação dependem do ritmo do colaborar, o método utilizado para medição do trabalho foi através do tempo padrão. Para chegar ao tempo padrão da operação, primeiro se faz necessário calcular o tempo médio preliminar da operação, que é soma aritmética das medições, dividido pela quantidade total de observações feitas.

Uma medição somente não é o suficiente para se determinar o tempo de uma atividade. É necessário que se façam algumas tomadas de tempo, mas quantas?

Nesta situação é necessário utilizar um cálculo de determinação de números de observações. De acordo com Wagner Cardoso 2017, a equação para a determinação do número de ciclos cronometrados, pode ser expressa através da equação 1, que adota nível de confiança de $95 \%$ e erro relativo de $5 \%$.

Equação 1 - Número de ciclos a serem cronometrados

$$
N^{\prime}=\left(\frac{40 \cdot \sqrt{\left(N * \Sigma X^{2}-(\Sigma X)^{2}\right.}}{\Sigma X}\right)^{2}
$$

Onde:

$\mathrm{N}^{`}=$ Número necessário de observações para prever o tempo verdadeiro com erro relativo de $5 \%$ e $95 \%$ de confiança

$\mathrm{N}=$ Número de observações feitas para o teste $\mathrm{X}=$ Duração do elemento $\quad \Sigma \mathrm{X}=$ Somatórias das leituras

Após a verificação do número de ciclos a serem cronometrados e se este for inferior ou igual ao número de cronometragens inicialmente executado, significa que a tomada de tempo é válida. Após esta validação, o próximo passo consiste na determinação do tempo normal para operação. 


\section{Otimização do processo produtivo de uma linha de panificação}

O tempo normal leva em conta a velocidade com que o colaborador está realizando a operação, e para tornar a medida válida para todos, é necessário a utilização de um fator para velocidade do trabalhador, com o objetivo de "normalizar" o trabalho. Para este estudo o fator de velocidade será de $100 \%$, um operador treinado, trabalhando em velocidade normal. O tempo normal pode ser expresso pela equação 2 (PEINADO, 2007).

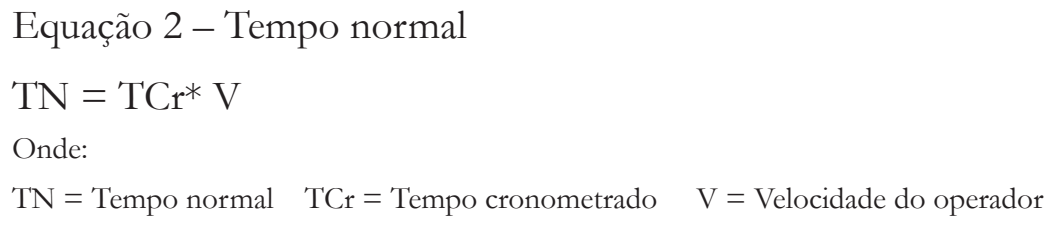

Um vez determinados o tempo normal, que é o tempo cronometrado ajustado a velocidade ou ritmo do operador, e validado a tomada de tempo através da equação 1, o próximo passo consiste da determinação do tempo padrão.

O tempo padrão é calculado multiplicando-se o tempo normal por um fator de tolerância, pois não é possível um trabalhador trabalhar o dia todo de forma ininterrupta, tanto por necessidades especiais como por motivos alheios a sua vontade. O fator de tolerância utilizado na empresa alvo do estudo é de 40 minutos por dia, levando em consideração que um dia de trabalho possui 480 minutos, o tempo total de tolerância é de $8 \%$. A equação para o tempo padrão pode ser expressa pela equação 3 (PEINADO, 2007): 


\section{Otimização do processo produtivo de uma linha de panificação}

Equação 3 - Tempo padrão

$\mathrm{TP}=\mathrm{TN} * \mathrm{FT}$

Onde:

$\mathrm{TN}=$ Tempo normal $\quad \mathrm{TP}=$ Tempo padrão $\quad \mathrm{FT}=$ Fator de tolerância

\subsubsection{Movimentação da matéria prima}

Esta etapa ocorre somente uma vez a cada turno. Cada movimentação abastece a produção com 50 sacos de trigo com $50 \mathrm{Kg}$ cada, totalizando $2.500 \mathrm{Kg}$ de trigo, e 12 misturas, matéria prima suficiente para a produção de um turno. Esta movimentação tem tempo padrão de 4 minutos, que divididos para um turno de $08 \mathrm{~h} 00 \mathrm{~min}$, compreende $0,1 \%$ de cada hora.

\subsection{Abastecimento da amassadeira}

Para medir o tempo de preparação de amassadeira o processo foi acompanhado por 35 vezes, em dias diferentes e horários alternados, com o objetivo de evitar vícios de medição. Os tempos verificados constam na tabela 1 a seguir. 


\section{Otimização do processo produtivo de uma linha de panificação}

Tabela 1 - Tempo de preparação da amassadeira em minutos

\begin{tabular}{|c|c|c|c|c|c|c|c|c|c|}
\hline $\mathbf{N}^{\circ}$ & Tempo & $\mathbf{N}^{\circ}$ & Tempo & $\mathbf{N}^{\circ}$ & Tempo & $\mathbf{N}^{\circ}$ & Tempo & $\mathbf{N}^{\circ}$ & Tempo \\
\hline 1 & 00:02:00 & 8 & 00:02:00 & 15 & 00:03:00 & 22 & 00:02:00 & 29 & $00: 02: 05$ \\
\hline 2 & 00:03:00 & 9 & 00:03:00 & 16 & 00:03:00 & 23 & 00:03:00 & 30 & 00:03:00 \\
\hline 3 & 00:03:00 & 10 & 00:02:00 & 17 & 00:03:00 & 24 & 00:02:00 & 31 & 00:02:00 \\
\hline 4 & 00:02:00 & 11 & 00:02:00 & 18 & 00:02:00 & 25 & 00:03:00 & 32 & 00:02:00 \\
\hline 5 & $00: 02: 00$ & 12 & $00: 02: 00$ & 19 & 00:03:00 & 26 & 00:02:00 & 33 & 00:02:00 \\
\hline 6 & $00: 02: 00$ & 13 & 00:03:00 & 20 & 00:03:00 & 27 & 00:03:00 & 34 & 00:03:00 \\
\hline 7 & 00:02:00 & 14 & 00:02:00 & 21 & 00:02:00 & 28 & 00:03:00 & 35 & $00: 02: 00$ \\
\hline
\end{tabular}

Fonte: Autores (2019)

Determinação do número de ciclos a serem cronometrados:

$$
\begin{aligned}
& n^{\prime}=\left(\frac{40 \cdot \sqrt{\left(N * \Sigma X^{2}-(\Sigma X)^{2}\right.}}{\Sigma X}\right)^{2} \\
& n^{\prime}=\left(\frac{1,96 \times 1}{0,05 \times 2,704 \times 2,33}\right)^{2}=22
\end{aligned}
$$

Constata-se que o número de tempos de ciclos necessários para que a amostragem seja confiável é de 22 . 


\section{Otimização do processo produtivo de uma linha de panificação}

Cálculo do tempo normal da operação.

$\mathrm{TN}=\mathrm{TCr} * \mathrm{~V}=183 \mathrm{~s} * 1,0=183 \mathrm{~s}$

Cálculo do tempo padrão da operação.

$\mathrm{TP}=\mathrm{TN} * \mathrm{FT}=183 \mathrm{~s} * 1,08=197 \mathrm{~s}$

\subsection{Amassamento da massa}

Para mensurar o tempo de batelada, o processo foi acompanhado durante 35 ciclos. O resultado pode ser verificado na tabela 2 .

Tabela 2 - Tempo de batelada em minutos

\begin{tabular}{|c|c|c|c|c|c|c|c|c|c|}
\hline $\mathbf{N}^{\circ}$ & Tempo & $\mathbf{N}^{\circ}$ & Tempo & $\mathbf{N}^{\circ}$ & Tempo & $\mathbf{N}^{\circ}$ & Tempo & $\mathbf{N}^{\circ}$ & Tempo \\
\hline 1 & $00: 23: 00$ & 8 & $00: 18: 00$ & 15 & $00: 24: 00$ & 22 & $00: 18: 00$ & 29 & $00: 26: 00$ \\
\hline 2 & $00: 21: 00$ & 9 & $00: 20: 00$ & 16 & $00: 23: 00$ & 23 & $00: 25: 00$ & 30 & $00: 21: 00$ \\
\hline 3 & $00: 22: 00$ & 10 & $00: 17: 00$ & 17 & $00: 25: 00$ & 24 & $00: 24: 00$ & 31 & 00:19:00 \\
\hline 4 & $00: 21: 00$ & 11 & $00: 28: 00$ & 18 & $00: 21: 00$ & 25 & $00: 24: 00$ & 32 & $00: 17: 00$ \\
\hline 5 & $00: 17: 00$ & 12 & $00: 21: 00$ & 19 & $00: 26: 00$ & 26 & 00:20:00 & 33 & 00:19:00 \\
\hline 6 & $00: 23: 00$ & 13 & $00: 24: 00$ & 20 & $00: 20: 00$ & 27 & $00: 24: 00$ & 34 & 00:19:00 \\
\hline 7 & $00: 23: 00$ & 14 & $00: 26: 00$ & 21 & $00: 24: 00$ & 28 & $00: 24: 00$ & 35 & 00:17:00 \\
\hline
\end{tabular}

Fonte: Autores (2019) 


\section{Otimização do processo produtivo de uma linha de panificação}

Por se tratar de uma etapa crítica para a produção, em que o tempo elevado de amassamento afeta diretamente a qualidade do produto em processo, foi necessária a construção de um gráfico de dispersão (figura 4), com o objetivo de visualizar o quanto os valores se afastam do tempo estabelecido para o processo.

Figura 4 - Tempo de batelada em minutos

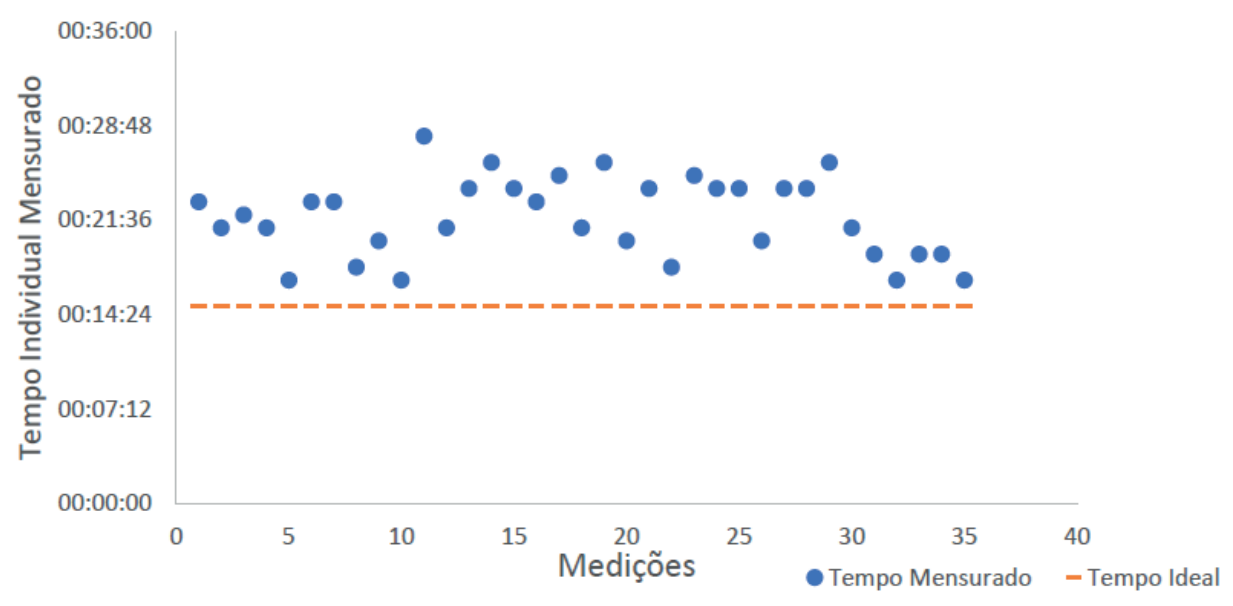

Fonte: Autores (2019).

Observa-se que o tempo do processo se encontra além do tempo ótimo de 15 minutos para o processo. Cada batelada possui $160 \mathrm{Kg}$ de massa, e cada pão tem peso de $0,055 \mathrm{Kg}$, logo cada batelada produz o equivalente a $160 \mathrm{Kg}$ divididos por $0,055 \mathrm{Kg}$, ou seja, 2.909 pães a cada 22 minutos, utilizando regra de 3 simples, 7.933 pães por hora. 


\section{Otimização do processo produtivo de uma linha de panificação}

\subsubsection{Cilindro}

Operação homem/máquina, possui grande demanda de energia por parte do operador. O tempo médio que uma batelada $(160 \mathrm{Kg})$ leva para ser cilindrada é de 15 minutos com a utilização de 2 operadores. Como uma massa é preparada a cada 21:50 minutos, sabe-se que durante 6:50 minutos os operadores não realizam de fato a tarefa de cilindrar a massa.

\subsubsection{Modelagem}

Para o cálculo de produção da modeladora foi utilizada a média da produção por hora feita pela máquina. Foram realizadas várias medições ao longo de 2 meses.

Para a modeladora de pães faz-se necessário dizer que a máquina possui capacidade máxima de produção especificada no manual de 12.000 pães/hora. Os resultados coletados podem ser conferidos na tabela 3 a seguir.

Tabela 3 - Produção da modeladora

\begin{tabular}{cccccc}
\hline $\mathbf{N}^{\circ}$ & Data & Pães/Hora & $\mathbf{N}^{\circ}$ & Data & Pães/Hora \\
\hline 1 & $03 /$ set & 7576 & 6 & $28 /$ set & 5567 \\
2 & $04 /$ set & 6677 & 7 & $11 /$ out & 3265 \\
3 & $14 /$ set & 7338 & 8 & $18 /$ out & 6413 \\
4 & $21 /$ set & 5932 & 9 & $19 /$ out & 7293 \\
5 & $26 /$ set & 5916 & 10 & $26 /$ out & 6693 \\
\hline Média Total & & & & &
\end{tabular}

Fonte: Autores (2019) 


\section{Otimização do processo produtivo de uma linha de panificação}

Observa-se que a média de produção/hora encontra-se aquém da capacidade máxima da máquina em uma taxa de 53\%. Dessa forma, conclui-se que durante um período de 60 minutos a máquina trabalha apenas 28 minutos. Pode-se mensurar a quantidade de carros feitos por hora, dividindo a quantidade de pães/hora pela quantidade de pães comportados por um carro, logo, teremos 5.857 pães/hora, divididos por 1.280 pães por carro, obtendo-se 4,5 carros por hora ou 1 carro cheio a cada 13 minutos.

\subsubsection{Separação}

A separação representa o processo em que um funcionário padrão treinado trabalha fazendo a coleta de pães diretamente da esteira modeladora. Para esta operação, dois operadores trabalham em conjunto. A cada ciclo o operador coleta 10 pães, 5 em cada mão. O tempo utilizado para encher uma forma com 80 pães foi mensurado, e o resultado encontra-se na tabela de dados a seguir.

Tabela 4 - Tempo para separação em segundos (80 pães)

\begin{tabular}{cccccccccc}
\hline $\mathbf{N}^{\circ}$ & Tempo & $\mathbf{N}^{\circ}$ & Tempo & $\mathbf{N}^{\mathbf{0}}$ & Tempo & $\mathbf{N}^{\mathbf{2}}$ & Tempo & $\mathbf{N}^{\circ}$ & Tempo \\
\hline 1 & 60 & 4 & 55 & 7 & 73 & 10 & 56 & 13 & 57 \\
2 & 60 & 5 & 51 & 8 & 50 & 11 & 67 & 14 & 51 \\
3 & 65 & 6 & 55 & 9 & 55 & 12 & 76 & 15 & 56 \\
\hline Média & & & & & & & & & $\mathbf{5 9 , 1 4}$
\end{tabular}

Fonte: Autores (2019). 


\section{Otimização do processo produtivo de uma linha de panificação}

Determinação do número de ciclos a serem cronometrados:

$$
\begin{aligned}
& n^{\prime}=\left(\frac{40 \cdot \sqrt{\left(N * \Sigma X^{2}-(\Sigma X)^{2}\right.}}{\Sigma X}\right)^{2} \\
& n^{`}=\left(\frac{40 \cdot \sqrt{\left(15 * 53317-(887)^{2}\right)}}{887}\right)^{2} \\
& n^{`}=27
\end{aligned}
$$

Constata-se que para os dados cronometrados, são necessárias 27 medições, frente às 15 realizadas, logo se faz necessário realizar mais 12 medições.

Tabela 5 - Tempo para separação em segundos (80 pães)

\begin{tabular}{cccccccc}
\hline $\mathbf{N}^{\circ}$ & Tempo & $\mathbf{N}^{\circ}$ & Tempo & $\mathbf{N}^{\circ}$ & Tempo & $\mathbf{N}^{\circ}$ & Tempo \\
\hline 1 & 56 & 4 & 60 & 7 & 57 & 10 & 66 \\
2 & 57 & 5 & 60 & 8 & 61 & 11 & 60 \\
3 & 61 & 6 & 57 & 9 & 60 & 12 & 57 \\
\hline Média & & & & & & & $\mathbf{5 9 , 3 4}$
\end{tabular}

Fonte: Autores (2019).

Após realizadas mais 12 tomadas de tempo, novamente verificou-se se o número de medições eram suficientes para o intervalo de confiança de $95 \%$. Para isso foram utilizados os dados das tabelas 4 e 5 . 


\section{Otimização do processo produtivo de uma linha de panificação}

$$
\begin{aligned}
& n^{`}=\left(\frac{40 \cdot \sqrt{\left(N * \Sigma X^{2}-(\Sigma X)^{2}\right.}}{\Sigma X}\right)^{-} \\
& n^{`}=\left(\frac{40 \cdot \sqrt{\left(27 * 95647-(1599)^{2}\right)}}{1599}\right)^{2} \\
& n^{`}=16
\end{aligned}
$$

Constata-se que o número de tempos de ciclos necessários para que a amostragem seja confiável é de 16, inferior as 27 medições realizadas.

Cálculo do tempo normal da operação.

$$
\mathrm{TN}=\mathrm{TCr} * \mathrm{~V}=59,3 \mathrm{~s} * 1.0=59,3 \mathrm{~s}
$$

Cálculo do tempo padrão da operação.

$$
\mathrm{TP}=\mathrm{TN} * \mathrm{FT}=59,3 \mathrm{~s} * 1,08=64,04 \mathrm{~s}
$$

Logo, dois operadores trabalhando em um ritmo normal possuem capacidade produtiva de 57 fôrmas por hora. Sabendo que cada forma é preenchida com 80 pães, conclui-se que a capacidade de produção para dois operadores é de 4.560 pães/hora. Nesta etapa do processo são utilizados 4 operadores, portanto a capacidade deste processo é de no máximo 9.120 pães/hora se não houver falhas de operação. 


\section{Otimização do processo produtivo de uma linha de panificação}

\subsubsection{Fermentação e crescimento}

Para o crescimento adequado dos pães são necessárias $1 \mathrm{~h} 45 \mathrm{~min}$ dentro da câmara de fermentação. A câmara possui espaço para até 15 carros, e para determinar a capacidade da câmara por hora, foi necessário verificar a taxa de entrada dos carros, pois por possuir tempo de operação estabelecido, a taxa de produção hora é igual a taxa de entrada dos carros, que é a taxa de saída do processo modelagem de 4,5 carros horas, ou 13 minutos para cada carro.

\subsubsection{Cocção}

O tempo necessário a cocção de um carro com 16 fôrmas é de 20 minutos. Sabendo que existem 4 fornos e tendo como premissa que todos os fornos estão funcionando com capacidade total, entende-se que a capacidade atual dos fornos é de $\mathrm{QF}^{*} \mathrm{CQ} \mathrm{QFF}^{*} \mathrm{QP}=15360$ pães/hora.

Onde:

QF: Quantidade de fornos $=4$

CQ: Capacidade de cocção por hora para cada forno $=3$ Carros

QF: Quantidade de formas por carro $=16$

QP: Quantidade de pães por forma $=80$

\subsubsection{Resfriamento}

$\mathrm{O}$ resfriamento consiste de duas etapas. Na primeira, o carro contendo as formas é resfriado com ventilação forçada, posteriormente o carro é levado a área de resfriamento natural, onde a equipe de expedição aguarda o pão chegar a temperatura de $30^{\circ} \mathrm{C}$, ideal para embalar os pães. Uma 


\section{Otimização do processo produtivo de uma linha de panificação}

temperatura acima de $30^{\circ} \mathrm{C}$ faz com que exista umidade dentro dos pacotes, criando ambiente ideal para proliferação de fungos e mofo.

Não foi possível determinar o tempo desta operação pois os operadores não têm nenhum controle ou tempo definido para esta etapa. Os carros ficam com ventilação forçada até que o operador em algum momento o movimente para área de resfriamento natural. Ao mesmo tempo, outros carros são transferidos diretamente do forno para área de resfriamento natural. Não existe critério ou método de trabalho definido.

\subsection{Determinação do gargalo produtivo}

Conhecidos os processos e as capacidades de produção atual, foi realizado o levantamento do gargalo de produção. Para facilitar a visualização e entendimento do processo, todos os tempos de produção média e ciclo foram convertidos para pães/hora.

- Movimentação de matéria prima: 4 minutos para $2.500 \mathrm{Kg}$ de trigo e 12 misturas, ou seja, 12 bateladas * 2.909 pães, produzindo 34.908 pães para 4 minutos de produção, ou 523.620 pães para 1 hora.

- Abastecimento da amassadeira: 3 minutos e 29 segundos minutos por receita de $160 \mathrm{Kg}$. Para uma hora temos 60 minutos divididos por 3.17 minutos, o que representa uma produção de 21 receitas de $160 \mathrm{Kg}$. Convertendo para pães por hora, chegamos a 61.090 pães para 1 hora.

- Amassamento da massa: 21.30 minutos para uma receita de $160 \mathrm{Kg}$, logo, para uma hora é possível fazer 2,79 bateladas de $160 \mathrm{Kg}$, que divididos por 0,055 Kg garantem produção de 8.116 pães para 1 hora. 


\section{Otimização do processo produtivo de uma linha de panificação}

- Cilindro: 15 minutos para $160 \mathrm{Kg}$ de massa com utilização de 2 operadores, logo, em 2 horas seria possível efetuar o processo de cilindrar 4 massas, ou seja, ((160 $\mathrm{Kg} * 4$ bateladas) $/ 0,055 \mathrm{Kg})=11.636$ pães/hora.

- Modelagem: Produção de 5.857 pães por hora, em média.

- Separação: Capacidade máxima para 4 operadores de 9.120 pães por hora.

- Fermentação: Produção de 5.857 pães por hora, em média.

- Forno: Capacidade produtiva máxima de 15.360 pães por hora

- Resfriamento: Para determinar a capacidade atual do processo de resfriamento foi necessário a realização de testes com tempos pré-determinados de permanência dos carros dentro da câmara de resfriamento. $\mathrm{O}$ teste que apresentou a melhor taxa de tempo-benefício foi de 20 minutos por carro. Dessa forma, ao longo de duas horas o pão atingiria a temperatura ótima de expedição. A câmara de resfriamento suporta simultaneamente 3 carros, logo a capacidade máxima pode ser definida por 3 carros * 3 turnos de resfriamento, como cada carro possui 1.280 pães, a capacidade é então definida por 3 carros $* 3$ turnos de resfriamento * 1.280 pães por carro, ou seja, 11.520 pães por hora.

Para sintetizar as informações, os valores foram agrupados na tabela 6. 


\section{Otimização do processo produtivo de uma linha de panificação}

Tabela 6 - Capacidade produtiva por recurso

\begin{tabular}{lr}
\hline Processo & Capacidade (Pães/Hr) \\
\hline Movimentação & 523.620 \\
Abastecimento & 61.090 \\
Amassamento & 8.116 \\
Cilindro & 11.636 \\
Modelagem & 5.857 \\
Separação & 9.600 \\
Forno & 15.360 \\
Fermentação & 5.857 \\
Resfriamento & 11.520 \\
\hline
\end{tabular}

Fonte: Autores (2019).

Verifica-se na tabela 6 que os processos onde se apresentam o gargalo produtivo são a modelagem e fermentação. Sabendo que a produção do processo de fermentação, depende da capacidade do processo de modelagem, conclui-se melhorando a capacidade de modelagem, podese também aumentar a produção do processo de fermentação.

\subsection{Análise dos gargalos}

A capacidade máxima do processo, não considerando as tarefas de movimentação e abastecimento, é de 11.636 pães por hora, limitados pela quantidade de cilindros existentes e também pela capacidade máxima da modeladora de 12.000 pães. Sendo assim, para aumentar a 


\section{Otimização do processo produtivo de uma linha de panificação}

produtividade e atingir esta quantidade, se faz necessário realizar melhorias em todos os processos com produtividade inferior a este número. Foram observados 4 processos onde a produtividade é inferior a 11.636, modelagem, amassamento, separação e fermentação.

\subsubsection{Modelagem}

O processo mais crítico e onde existe a maior perda de produtividade em relação a capacidade de produção é a modelagem. Para facilitar o acompanhamento das paradas foram criadas siglas referentes a cada parada anteriormente identificada:

FMM - Falta de massa (Motivo masseira); FOF - Parada por falta de operador para forma; FER - Parada por fermentação; TF/ST - Parada para troca de faca; FOC - Parada para troca de carro; FOG - Falta de operador para alimentação da modeladora (GA); FAGA - Falta de alimentação para modeladora (GA); CNC - Massa não conforme (Cilindro); FMC - Falta de massa (Motivo Cilindro); LPGA - Parada para limpeza da esteira modeladora (GA); MS - Máquina suja; RU - Reunião.

Foram realizadas 8 horas de observações, o resultado das medições pode ser conferido na figura 5 , a seguir. 


\section{Otimização do processo produtivo de uma linha de panificação}

Figura 5 - Gráfico de pareto tempo de parada

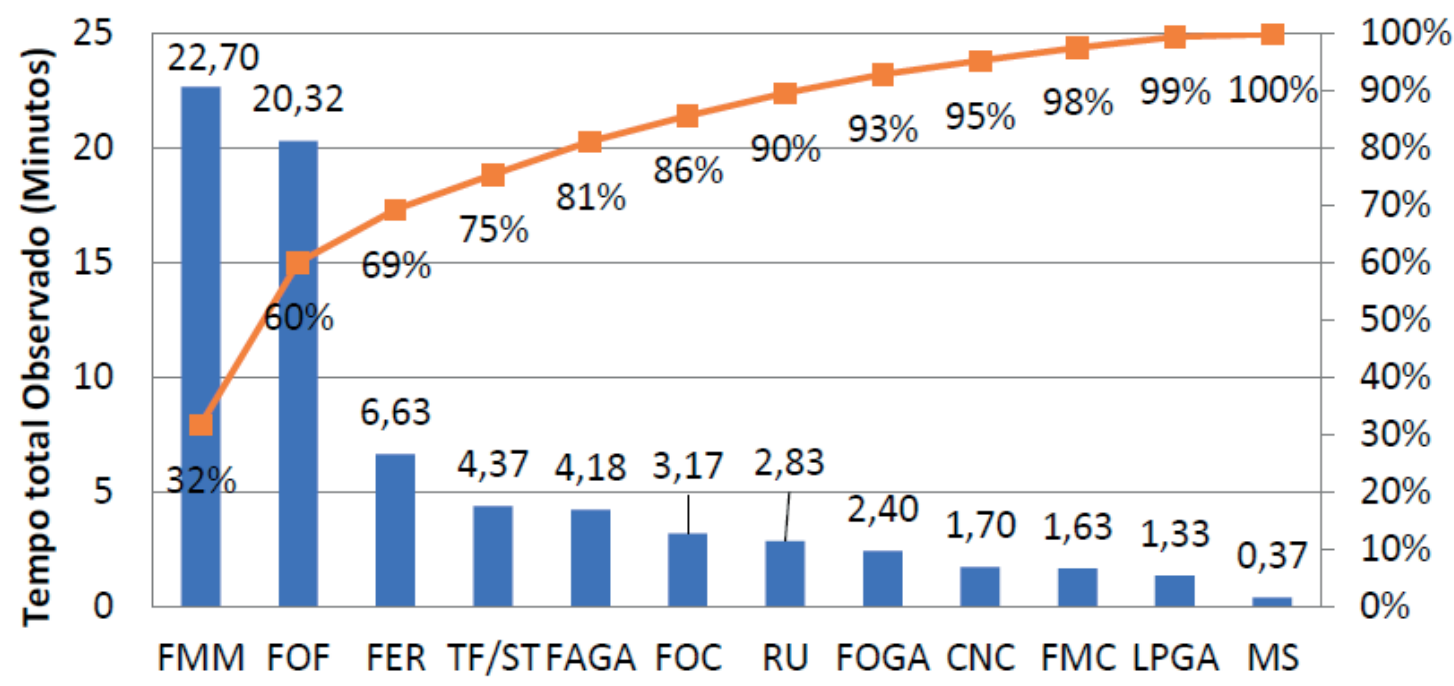

Fonte: Autores (2019).

É possível observar no gráfico que $70 \%$ do tempo perdido ocorre devido apenas 3 fatores.

1. FMM: Falta de massa por motivo de masseira;

2. FOF: Falta de operador para forma (tempo em que a máquina para devido à alta velocidade da esteira);

3. FER: Fermentação antecipada. 


\section{Otimização do processo produtivo de uma linha de panificação}

Analisando a situação, é possível perceber que os motivos 1 e 3 advém do mesmo processo e tem causa comum aos dois, que é o tempo em que a massa fica na amassadeira, um tempo acima de 15 minutos ocasiona falta de massa para alimentar a máquina modeladora, assim como ocasiona a fermentação antecipada da massa, sendo necessário seu descarte, ocasionando então a parada de máquina até que a próxima batelada seja concluída.

O motivo 2, falta de operador/forma, representa o momento em que a esteira para devido à não capacidade dos operadores de selecionar o pão com a esteira em funcionamento.

A produção foi acompanhada por um período de 8 horas e deste tempo retira- se o tempo de startup inicial, conforme será mencionado a seguir (Tabela 7).

Pode-se dizer dessa forma que a máquina foi acompanhada durante 416 minutos, se foram observados 71 minutos de parada, conclui-se que durante 17\% (71 minutos / 416 minutos) do tempo a máquina é improdutiva.

Outra perda identificada durante as medições foi o tempo necessário ao funcionamento da máquina em cada início de turno e volta de intervalos. 


\section{Otimização do processo produtivo de uma linha de panificação}

Tabela 7 - Tempo de Start-up

\begin{tabular}{ccccc}
\hline & & TEMPO DO START-UP ATÉ 1 & PÃO & \\
$\mathbf{N}^{\mathbf{0}}$ & ÍNíCIO & $\mathbf{1}^{\circ}$ PÃO & TEMPO DE ESPEA (Min) & MOTIVO \\
\hline 1 & $07: 00$ & $07: 41$ & $00: 41$ & Aguardando Masseira \\
2 & $07: 00$ & $07: 32$ & $00: 32$ & Aguardando Masseira \\
3 & $07: 00$ & $07: 44$ & $00: 44$ & Aguardando Masseira \\
4 & $07: 00$ & $07: 47$ & $00: 47$ & Aguardando Masseira \\
5 & $12: 30$ & $12: 54$ & $00: 24$ & Aguardando Masseira \\
6 & $12: 45$ & $13: 05$ & $00: 20$ & Aguardando Masseira \\
7 & $07: 00$ & $07: 42$ & $00: 42$ & Aguardando Masseira \\
8 & $12: 30$ & $13: 00$ & $00: 30$ & Aguardando Masseira \\
9 & $12: 30$ & $12: 57$ & $00: 27$ & Aguardando Masseira \\
10 & $17: 00$ & $17: 30$ & $00: 30$ & Aguardando Masseira \\
11 & $17: 00$ & $17: 29$ & $00: 29$ & Aguardando Masseira \\
12 & $17: 00$ & $17: 32$ & $00: 32$ & Aguardando Masseira \\
13 & $17: 00$ & $17: 25$ & $00: 25$ & Aguardando Masseira \\
\hline Total & & & & 07:03 \\
Média & & & & 00:32
\end{tabular}

Fonte: Autores (2019).

Para cada turno ou volta de intervalo existe em média um desperdício de 32 minutos, somado os 2 turnos, a máquina é improdutiva durante 64 minutos em um turno de 8 horas (480 minutos). Dividindo a quantidade de tempo perdida de 64 minutos, pela quantidade de tempo disponível de 480 minutos, chega-se a perda de $13,3 \%$ por hora de máquina disponível. 


\section{Otimização do processo produtivo de uma linha de panificação}

\subsubsection{Retrabalho}

Durante as medições do tempo de parada, percebe-se que existia uma grande quantidade de retrabalho ou pães não conformes. Para o acompanhamento, foram mensuradas a quantidade de pães não conformes na unidade de $\mathrm{Kg} / \mathrm{h}$. Ou seja, foram feitos acompanhamentos de hora em hora e mensurados quantos $\mathrm{Kg}$ por hora foram retrabalhados de acordo com a quantidade de massa produzida. Desta forma verificou-se que, em média, existe 16\% de retrabalho para cada hora produzida. Para esta situação pode-se dizer que retrabalho é sinônimo de máquina parada, pois o tempo necessário para refazer os pães não conformes, é também o mesmo tempo que não seria desperdiçado caso o pão estivesse bom no primeiro momento. O tempo total de parada, somados todos os desperdícios é de $46 \%$ por hora, ou 24 minutos.

\subsubsection{Amassamento}

O amassamento da massa, conforme mensurado anteriormente dura em média 21 minutos, o que representa 2,85 bateladas por hora. O tempo ideal para amassamento é de 15 minutos, o que representa 4 bateladas por hora, um aumento de $29 \%$ de produção, que passaria de 8.290 pães por hora para 11.636 pães por hora.

\subsubsection{Separação}

O processo de separação conta com 4 funcionários e o tempo padrão para o processo é conhecido, são 59:52 segundos para 160 pães com utilização de 4 operadores. Em uma hora isso representa 9.600 pães hora, como já mencionado anteriormente. A esteira alimentadora possui capacidade para até 8 operadores, o que representaria 19.200 pães hora, porem para o processo são necessários 11.636 pães hora, ou 6 operadores. 


\section{Otimização do processo produtivo de uma linha de panificação}

\subsection{Sugestões de melhoria}

Com base no estudo realizado, as sugestões de melhorias têm como objetivo sanar os problemas, aumentar a produtividade e diminuir os custos de operação. Através dos cálculos, pode-se observar que é possível produzir a demanda diária de 70.000 pães em apenas um turno, devendo ser fabricados 8.750 pães por hora. Para que isso ocorra, são necessárias algumas ações:

a. Padronização do tempo de amassamento para 15 minutos.

b. Entrada de 1 operador com 1 hora de antecedência, este ficará responsável por realizar a movimentação do material até a área de produção e por bater a primeira massa, dessa forma a equipe de produção não irá ficar ociosa aguardando a massa ficar pronta, eliminando 32 minutos de espera em cada reinicio de produção.

c. Alteração da produção toda para apenas 1 turno, eliminando 8 colaboradores e 1 turno, diminuindo a quantidade total de 20 para 12 colaboradores, diminuição de 40\%.

d. Adição de 2 operadores para o processo de seleção, aumentando a capacidade produtiva de 9.600 pães hora para 12.000 pães hora.

\subsection{Resultados pós testes}

As sugestões de melhorias foram colocadas em prática por 1 semana e os resultados foram satisfatórios. A produção média por hora aumentou de forma significativa, passando de 6.267 pães por hora para 11.500 pães por hora e toda a demanda foi produzida em apenas 8 horas.

Anteriormente eram fabricados aproximadamente 85.000 pães por HomemXMês. Com as melhorias instaladas este número passou para 141.666 pães por HomemXMês. Somente um turno 


\section{Otimização do processo produtivo de uma linha de panificação}

de trabalho foi o suficiente para produção total da demanda. O objetivo inicial de aumentar a produtividade do equipamento para $85 \%$ foi alcançado com sucesso, chegando a $95 \%$, um aumento de $42 \%$ se comparado aos $53 \%$ mensurados neste estudo.

A figura 6 representa o turno de trabalho adotado durante o período de testes. Anteriormente eram utilizadas 2 equipes de trabalho e cada equipe contava com 10 colaboradores. Após as mudanças realizadas foi possível reduzir 1 equipe e 8 colaboradores.

Figura 6 - Distribuição de jornada e postos de trabalho

\begin{tabular}{|c|c|c|c|c|c|c|c|c|c|c|c|c|c|}
\hline Op & 06 às 07 & 07 às 08 & 08 ás 09 & 09 às 10 & 10 às 11 & 11 às $11: 30$ & $11: 30$ às 12 & 12 às $12: 30$ & $12: 30$ às 13 & 13 às 14 & 14 às 15 & 15 às 16 & 16 às 17 \\
\hline 1 & Massa & Massa & Massa & Massa & Massa & Inter & Inter & Massa & Massa & Massa & Massa & & \\
\hline 2 & Forma & Forma & Forma & Forma & Forma & Massa & Inter & Inter & Forma & Forma & Forma & & \\
\hline 3 & & AGA & AGA & AGA & AGA & AGA & Inter & Inter & AGA & AGA & AGA & AGA & \\
\hline 4 & & CILINDRO & CILINDRO & CILINDRO & CILINDRO & CILINDRO & Inter & Inter & CILINDRO & CILINDRO & CILINDRO & CILINDRO & \\
\hline 5 & & CILINDRO & CILINDRO & CILINDRO & CILINDRO & CILINDRO & Inter & Inter & CILINDRO & CILINDRO & CILINDRO & CILINDRO & \\
\hline 6 & & $\mathrm{Ga}$ & $\mathrm{Ga}$ & $\mathrm{Ga}$ & $\mathrm{Ga}$ & $\mathrm{Ga}$ & Inter & Inter & $\mathrm{Ga}$ & $\mathrm{Ga}$ & $\mathrm{Ga}$ & Ga & \\
\hline 7 & & Ga & $\mathrm{Ga}$ & $\mathrm{Ga}$ & Ga & Ga & Inter & Inter & $\mathrm{Ga}$ & $\mathrm{Ga}$ & $\mathrm{Ga}$ & $\mathrm{Ga}$ & \\
\hline 8 & & Ga & $\mathrm{Ga}$ & $\mathrm{Ga}$ & Ga & Ga & Inter & Inter & $\mathrm{Ga}$ & $\mathrm{Ga}$ & $\mathrm{Ga}$ & $\mathrm{Ga}$ & \\
\hline 9 & & $\mathrm{Ga}$ & $\mathrm{Ga}$ & $\mathrm{Ga}$ & $\mathrm{Ga}$ & $\mathrm{Ga}$ & Inter & Inter & $\mathrm{Ga}$ & $\mathrm{Ga}$ & $\mathrm{Ga}$ & $\mathrm{Ga}$ & \\
\hline 10 & & $\mathrm{Ga}$ & $\mathrm{Ga}$ & $\mathrm{Ga}$ & $\mathrm{Ga}$ & $\mathrm{Ga}$ & Inter & Inter & $\mathrm{Ga}$ & $\mathrm{Ga}$ & $\mathrm{Ga}$ & $\mathrm{Ga}$ & \\
\hline 11 & & Ga & $\mathrm{Ga}$ & $\mathrm{Ga}$ & $\mathrm{Ga}$ & $\mathrm{Ga}$ & Inter & Inter & Ga & $\mathrm{Ga}$ & $\mathrm{Ga}$ & $\mathrm{Ga}$ & \\
\hline 12 & & & & & Forno & Forno & Forno & Forno & Forno & Inter & Forno & Forno & Forno \\
\hline
\end{tabular}

Fonte: Autores (2019). 


\section{Otimização do processo produtivo de uma linha de panificação}

\section{Conclusões}

A produção tem papel fundamental dentro das empresas, e necessita cada vez mais de planejamento estratégico para atingir os melhores indicadores possíveis.

O setor de panificação é responsável por produzir todos os panificados necessários para operação e atendimento aos clientes, aproximadamente 1.700 .000 pães por mês, mais significativa quantidade de bolos.

O objetivo principal deste estudo foi analisar os processos atuais e sugerir mudanças no método de trabalho e gestão da produção.

Com o levantamento e análise dos dados foi possível avaliar vários pontos de melhoria a serem aperfeiçoados. A partir desta análise foram sugeridas mudanças de imediata implantação e que não refletiam custos operacionais nem de implementação. Durante a fase de levantamento de dados encontrou-se grande dificuldade em levantar as informações, em parte devido à pouca experiência e pela grande mescla de diferentes atividades realizadas para produção. O processo de adequação e balanceamento da produção exige minuciosa avaliação dos processos, pois informações erradas podem levar a tomadas de decisões equivocadas por partes dos gestores de produção.

Após aplicadas as mudanças sugeridas, constatou-se grande aumento de produtividade e maior facilidade de gestão. O turno noturno foi eliminado, facilitando a solução de problemas que possam aparecer. Além de todas as melhorias e ganhos antes citados, não foram realizados investimentos para as mudanças realizadas. 


\section{Otimização do processo produtivo de uma linha de panificação}

\section{Referências}

ANTUNES, J. Sistemas de produção: conceitos e práticas para projeto e gestão da produção enxuta. Porto Alegre: Bookman, 2008.

CARDOSO, W. Engenharia de métodos e produtividade: A teoria na prática. 1 ed. Ananindeua: Itacaiúnas, 2018.

CHIAVEnATO, I. Administração Geral e Pública. Rio de Janeiro: Elsevier, 2006.

FITZSIMONS, J. A; FITZSIMONS, M. J. Administração de serviços: Operações, estratégias e tecnologias. $4^{\mathrm{a}}$ edição, Porto Alegre: Bookman, 2004.

HANSEN, R. C. Eficiência global dos equipamentos: uma poderosa ferramenta de produção/manutenção para o aumento dos lucros. Porto Alegre: Bookman, 2006.

MARCONI, M. A.; LAKATOS, E. V. Fundamentos de metodologia científica. 6 ed., São Paulo: Editora Atlas, 2006.

MOREIRA, D. A. Administração da Produção e Operações. 3. ed. São Paulo: Pioneira, 1998.

OLIVEIRA, S. E.; ALLORA, V.; SAKAMOTO, F. T. C. Utilização conjunta do método UP’ (Unidade de Produção -UEP') com o Diagrama de Pareto para identificar as oportunidades de melhoria dos processos de fabricação: um estudo na agroindústria de abate de frango. 2006. Custos e Agronegócio, v. 2 - n.2 2006. 


\section{Otimização do processo produtivo de uma linha de panificação}

PEINADO, J. Administração da Produção - Operações Industriais e de Serviços. Curitiba: UnicenP, 2007.

SLACK, N. Administração da Produção. São Paulo: Atlas, 1997.

TUBINO, D. F. O Planejamento e Controle da Produção - Teoria e Prática. São Paulo: Atlas, 2007.

WERKEMA, M. C. C. Ferramentas estatísticas básicas para o gerenciamento de processos. Belo Horizonte: Fundação Cristiano Ottoni, 1995.

Submetido em 04 de maio de 2020

Aceito em 14 de junho de 2020

Publicado em 18 de dezembro de 2020 